\title{
Molecular radiotheranostics for neuroendocrine tumours
}

\author{
Authors: Shaunak Navalkissoor, ${ }^{A}$ Glenn Flux ${ }^{B}$ and Jamshed Bomanji
}

This article discusses the important role of nuclear medicine imaging and therapy in the management of neuroendocrine tumours (NETs). Somatostatin receptor scintigraphy has a high impact on patient management versus conventional imaging. Molecular radiotherapy is an important part of the management of patients with NETs. Selection of patients for molecular radiotherapy in NETs is based on uptake on their radionuclide imaging study. The imaging agent has the same mechanism of uptake as the therapeutic agent. Thus, the imaging study preselects patients that are likely to concentrate radiation within their tumours.

\section{Introduction}

Neuroendocrine tumours (NETs) represent a spectrum of tumours that can arise from various parts of the body and have variability in clinical presentation. They are often classified by embryonic origin into foregut, midgut and hindgut tumours. Foregut tumours develop in the respiratory tract, thymus, stomach, duodenum and pancreas; midgut tumours develop in the small bowel, appendix, caecum and ascending colon; hindgut tumours develop in the transverse colon, descending colon, sigmoid colon or rectum. ${ }^{1}$ NETs have a spectrum of presentations, ranging from well differentiated to poorly differentiated tumours, functioning to non-functioning and have variable proliferative activity. ${ }^{2}$ The tumour differentiation, grading (based on tumour proliferation and mitotic activity) and the site of primary tumour are important factors in making therapeutic decisions in management of NET patients.

Unlike many cancers, large proportions of NETs tend to be slow growing and are not associated with increased metabolic activity. These are therefore not visualised on fludeoxyglucose (FDG) positron emission tomography/ computerised tomography (PET/CT). Other functional imaging techniques have thus been employed to stage NETs and determine suitability for molecular radiotherapy. Despite variability in presentation, NETs have similar properties in that the majority of well-differentiated and some high-grade tumours can concentrate neuroamines and have a high degree

Authors: ${ }^{\text {A }}$ consultant in nuclear medicine, Royal Free London NHS Foundation Trust, London, UK; ${ }^{B}$ head of radio-isotope physics, Royal Marsden Hospital \& Institute of Cancer Research, London, UK; ${ }^{C}$ professor and head of clinical nuclear medicine, University College London Hospitals NHS Foundation Trust, London, UK of somatostatin receptor (SSR) expression. This high degree of SSR expression and neuroamine concentration can be exploited both in terms of imaging and therapy with radiolabelled somatostatin analogues and radiolabelled catecholamine analogue meta-iodobenzyl guanidine (MIBG). ${ }^{3,4}$

Selection of patients for molecular radiotherapy is based on uptake on their radionuclide imaging study. In nuclear medicine, theranostics is a combination of a diagnostic agent and a therapeutic agent where the target imaged/treated is the same. The imaging agent has the same mechanism of uptake as the therapeutic agent. Thus, the imaging study pre-selects patients that are likely to concentrate radiation within their tumours. The imaging and therapeutic radiopharmaceutical forms a theranostic pair. This article discusses the important role of radionculide imaging and radionculide therapy in the management of NETs based on these mechanisms. An algorithm of the management of NETs is shown in Fig 1, with the lower left box highlighting the role of radiolabelled SSR and MIBG in the management of NETs. FDG PET/CT may be useful in predicting response to peptide receptor radionuclide therapy (PRRT) and in determining progression-free survival with those with FDG positive well differentiated having worse survival.

\section{Key points}

Somatostatin receptor scintigraphy plays an important role in the management of NETs, providing more accurate staging and determining whether patients are eligible for cold or radiolabelled somatostatin analogues

PRRT is an effective treatment in well-differentiated NETs with limited toxicity

MIBG imaging is a well established imaging modality for catecholamine secreting neuroectodermal tumours

\footnotetext{
${ }^{131}$ I-MIBG therapy is an effective treatment in metastatic neuroectodermal tumours, but has generally been replaced by PRRT in NETs

Access to PRRT varies across the UK

KEYWORDS: ${ }^{177} \mathrm{Lu},{ }^{90} \mathrm{Y}, \mathrm{MIBG}$, PRRT, somatostatin receptor scintigraphy
} 


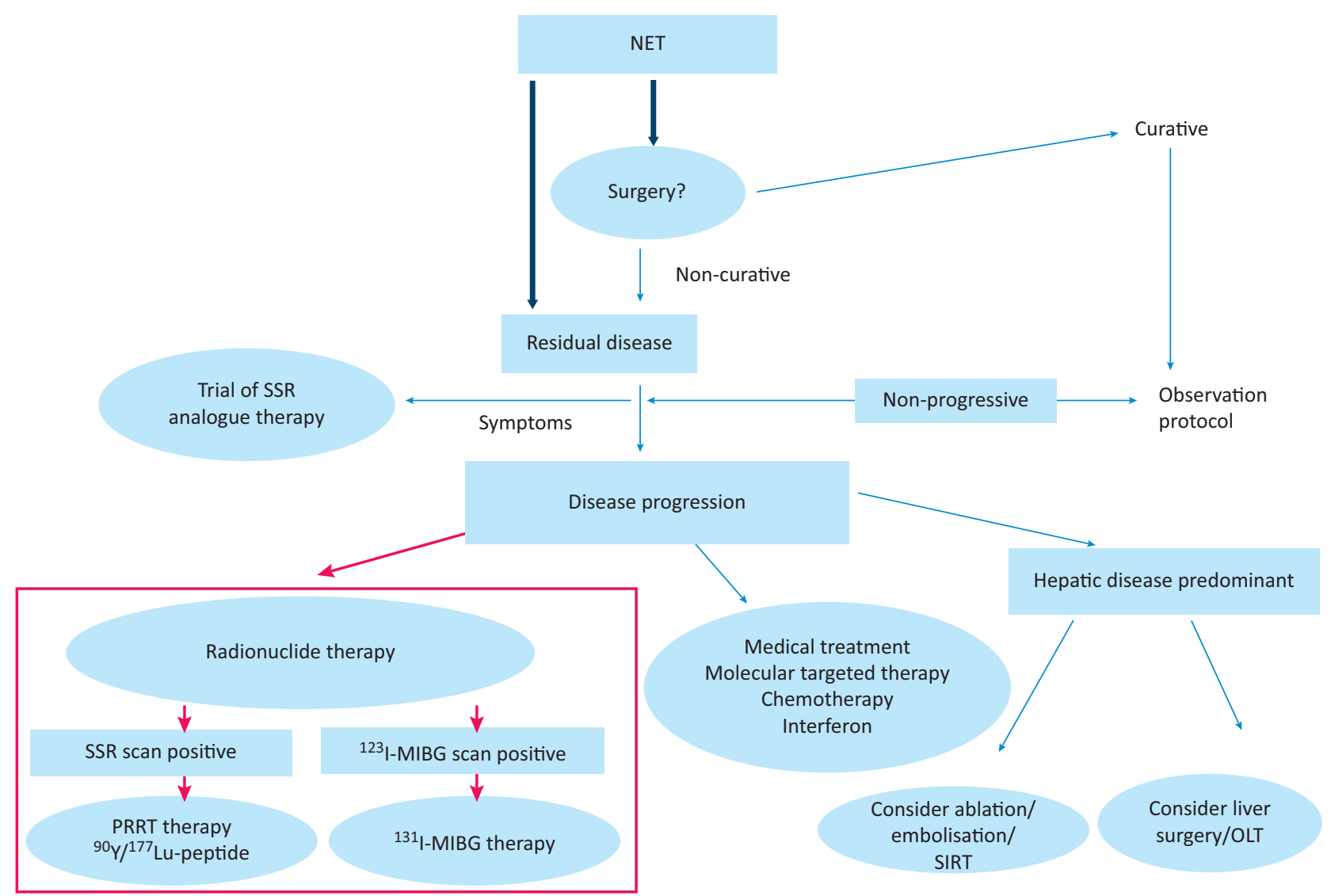

Fig 1. An algorithm of the therapeutic options in NETs based on the United Kingdom and Ireland Neuroendocrine Tumour Society (UKINETS) and Royal Free Algorithm. ${ }^{123} \mathrm{I}=$ Iodine-123; ${ }^{177} \mathrm{Lu}=$ Lutetium-177; ${ }^{90} \mathrm{Y}=$ Yttrium-90; MIBG = meta-iodobenzyl guanidine; NET = neuroendocrine tumour; OLT = orthotopic liver transplantation; PRRT = peptide receptor radionuclide therapy; SIRT = selective internal radiation therapy; SSR = somatostatin receptor;

\section{Somatostatin receptor scintigraphy}

SSR imaging has a major role to play in imaging of NETs and is now integral to their management, allowing more accurate staging and demonstrating suitability for therapies ('cold' or radiolabelled somatostatin analogues). ${ }^{5}$ SSRs are expressed in a number of normal cells, including the pituitary gland, thyroid, spleen and kidney. In addition, several tumours have been found to express SSRs. In particular, NETs have been shown to have a high incidence and density of receptors. ${ }^{6}$ Five SSR subtypes have been identified with subtype-2 the most overexpressed receptor in NETs.

Somatostatin receptor scintigraphy was first introduced in the late 1980s and subsequently there have been many variations in the radiopharmaceuticals used to image the SSR. ${ }^{7}$ There are two components to the SSR analogue: the radionuclide and the somatostatin analogue peptide, linked by a chelator.

\section{Single photon emission computerised tomography gamma camera somatostatin receptor scintigraphy}

The first somatostatin analogue used for imaging of NETs was octreotide and this remains as one of the most popular analogues for imaging NETs. Octreotide labelled with Indium-111 $\left({ }^{111} \mathrm{In}\right)$ is commercially available as Octreoscan ${ }^{\circledR}$ and remains the most popular SSR imaging agent. ${ }^{111}$ In has a half-life of 68 hours and delayed imaging (24-48 hours) is usually required to ensure reduction in background activity caused by clearance via the renal and hepatobiliary system. Labelling of somatostatin analogues with the most commonly used radionuclide in nuclear medicine ${ }^{99 \mathrm{~m}}$ Technetium $\left({ }^{99 \mathrm{~m}} \mathrm{Tc}\right)$ has also been achieved, allowing single day imaging. ${ }^{8}$

Uptake of Octreoscan ${ }^{\circledR}$ and ${ }^{99 \mathrm{~m}} \mathrm{Tc}$-HYNIC-peptides is seen in the majority of patients (>75\%) with NETs; major exceptions include poorly differentiated NETs (because of a lower expression of SSR-2) and insulinomas with a reduced sensitivity of $50-70 \%$ (probably because of small tumour size or poor expression of SSR-2 receptor). ${ }^{9}$

\section{PET somatostatin receptor scintigraphy}

The development of a generator producing PET radionuclide gallium-68 $\left({ }^{68} \mathrm{Ga}\right)$ has been the biggest evolution in SSR imaging over the past decade, becoming increasingly more popular in centres where PET/CT imaging is available. ${ }^{68} \mathrm{Ga}$ has a convenient physical half-life of 68 minutes and decays by positron emission, giving the advantages of increased sensitivity and resolution that modern PET imaging allows. It is produced by a germanium- $68\left({ }^{68} \mathrm{Ge}\right)-{ }^{68} \mathrm{Ga}$ generator that 
Table 1. Studies evaluating the efficacy of ${ }^{177}$ Lu-DOTATATE

\begin{tabular}{|c|c|c|c|c|c|c|}
\hline Study & Patients, $n$ & Location & CR/PR & PD & Time to progress, months & Median OS, months \\
\hline Kwekkemboom et al ${ }^{18}$ & $310(504)$ & Rotterdam & $91 / 301(30 \%)$ & $61 / 310(20 \%)$ & 33 & 46 \\
\hline Pencharz et al ${ }^{19}$ & 79 & London & $10 / 79(13 \%)$ & $18(23 \%)$ & 28 & - \\
\hline $\begin{array}{l}\text { Claringbold et al (plus } \\
\text { capecitabine) }^{23}\end{array}$ & 33 & Perth & $24 \%$ & $6 \%$ & & $88 \%$ at 24 months \\
\hline NETTER-1 study ${ }^{20}$ & 116 & $\begin{array}{l}\text { Multicentre: } \\
\text { Europe and } \\
\text { USA }\end{array}$ & $18 \%$ & $5 \%$ & 40 months estimate & \\
\hline
\end{tabular}

lasts $7-12$ months and can be eluted (to produce ${ }^{68} \mathrm{Ga}$ ) twice a day. The ${ }^{68} \mathrm{Ga}$ radiopeptide study is completed within $2-3$ hours of administration of tracer. In addition, the patient effective radiation dose is less than half that of ${ }^{111}$ In-DTPA-octreotide. ${ }^{10}$

${ }^{68} \mathrm{Ga}$ has been linked with three main somatostatin analogues (via linker DOTA): DOTA-TATE, DOTA-NOC and DOTATOC. ${ }^{11}$ The main differences between these compounds are small changes in the peptide side chain, which result in different affinities to the five SSR subtypes.

There is no doubt that ${ }^{68} \mathrm{Ga}$-DOTA-peptides represent a significant evolution in SSR imaging over ${ }^{111}$ In-DTPAoctreotide imaging. In a study, it was shown that ${ }^{68} \mathrm{Ga}-$ DOTA-TATE changed the management in 36 out of the 51 (70\%) patients who had either no uptake (35 patients) or low-grade uptake (15 patients) of ${ }^{111}$ In-octreotide. ${ }^{12}$ A recent meta-analysis demonstrated a pooled sensitivity of $93 \%$ and specificity of $96 \%$ in staging well-differentiated NETs with an impact in management of approximately $50 \% .^{13}$

\section{Peptide receptor radionuclide therapy}

PRRT is the term commonly used to describe treatment with $\beta$-emitting radiolabelled somatostatin analogues: the peptide receptor being the SSR. PRRT has been performed with various somatostatin analogues labelled with ${ }^{111} \mathrm{In}$, Yttrium-90 $\left({ }^{90} \mathrm{Y}\right)$ and Lutetium-177 $\left({ }^{177} \mathrm{Lu}\right)$. From these, three main agents have developed:

$$
\begin{aligned}
& 1{ }^{90} \text { Y-DOTA octreotide }\left({ }^{90} \text { Y-DOTATOC }\right) \\
& 2{ }^{90} \text { Y-DOTA octreotate }\left({ }^{90} \text { Y-DOTATATE }\right) \\
& 3{ }^{177} \text { Lu-DOTA octreotate }\left({ }^{177} \text { Lu-DOTATATE }\right) .
\end{aligned}
$$

The radionuclides involved have different physical characteristics, which may have a bearing on both their efficacy and toxicity, ie ${ }^{90} \mathrm{Y}$ has a higher energy beta particle emission than ${ }^{177} \mathrm{Lu}$, and thus may be suited to treating larger tumour masses but has more toxicity.

\section{${ }^{90} Y$-DOTATATE/DOTATOC anti-tumour effects}

Partial response or disease stabilisation has been reported between 70 and $80 \% \cdot{ }^{14-17}$ In addition, the majority of studies have shown excellent symptomatic response in $>70 \%$ of patients. Time to progression in reported studies ranged from 13-29 months.

Toxicity

The main toxicities of ${ }^{90} \mathrm{Y}$-PRRT are myelosuppression and renal impairment. The rate of permanent renal toxicity showed variation (range 1-9\%). The greatest recorded significant permanent renal toxicity is quite high at $9 \%$ from a Swiss study of over 1,000 patients. ${ }^{14}$ The predictors for severe renal toxicity by the Swiss study included advancing age, baseline glomerular filtration rate and high uptake of tracer by the kidneys on the baseline Octreoscan ${ }^{\circledR}$.

Bone marrow toxicity is seen in approximately $12 \%$ of patients overall and is usually transient, presenting as either thrombocytopenia or leucopenia. Mild to moderate liver toxicity has also been recorded in $1 \%$ of patients all of whom had extensive bi-lobar liver metastases.

\section{${ }^{177}$ Lu-PRRT anti-tumour effects}

Several studies have shown that ${ }^{177} \mathrm{Lu}$-PRRT is a useful treatment and it has now become the most popular form of PRRT because of its efficacy and low toxicity (Table 1). ${ }^{18-21}$ The most notable published study is a recent phase III multicentre international study (NETTER-1) that is the first randomised controlled trial in PRRT. This trial has completed recruitment and was published in early $2017 .^{20}$ This is a randomised comparator controlled study comparing standard activity of ${ }^{177} \mathrm{Lu}$-DOTATATE with high dose octreotide LAR $60 \mathrm{mg}$ (which is a non-radioactive somatostatin analogue used in the management of NET patients, Fig 1) in patients with inoperable, progressive, SSR positive, midgut carcinoid tumours. The results have been extremely promising, demonstrating a progression-free survival of approximately 40 months versus 8.4 months for octreotide LAR. This appears to be far superior to other systemic treatment modalities for metastatic disease in NETs. An example of a patient with a positive ${ }^{68} \mathrm{Ga}$-DOTATATE study who went on to have ${ }^{177} \mathrm{Lu}$ DOTATATE therapy is demonstrated in Fig 2. A patient with a partial response to ${ }^{177} \mathrm{Lu}$-DOTATATE therapy is shown in Fig 3.

Toxicity

Data from the largest reporting centre demonstrated WHO Grade 3/4 haematological toxicity in $9.4 \%$ of 504 patients. $^{18}$ Of these, two patients $(0.4 \%)$ developed renal insufficiency. There were three patients with serious liver toxicity. One of these patients (with diffuse liver metastases) died of hepatic failure. Four patients developed myelodysplastic syndrome, one of which was likely to be related to previous alkylating chemotherapy. Similarly NETTER-1 data showed that $<10 \%$ of patients developed myelosuppression. 
A

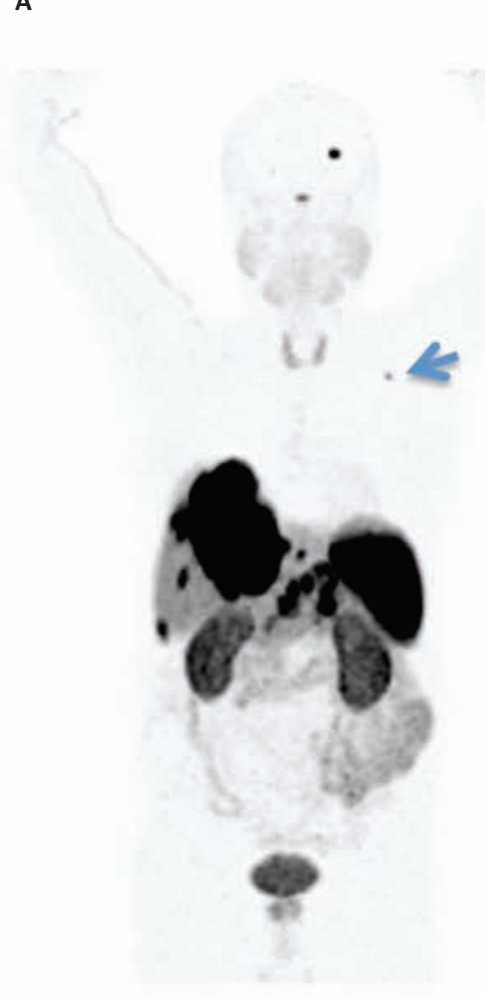

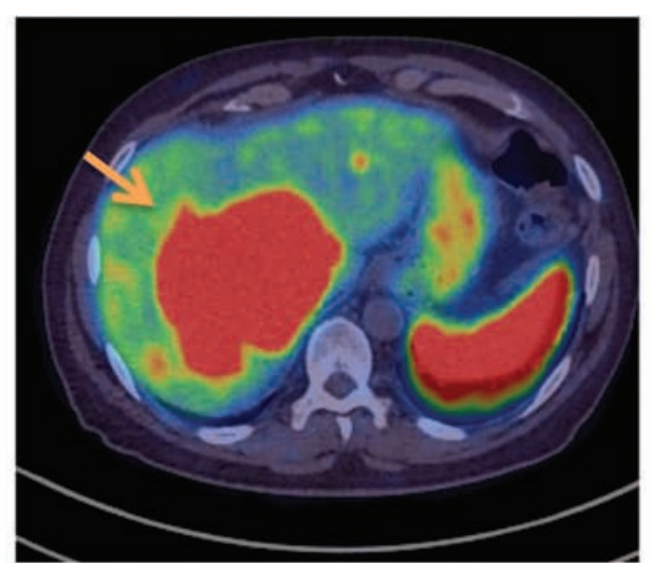

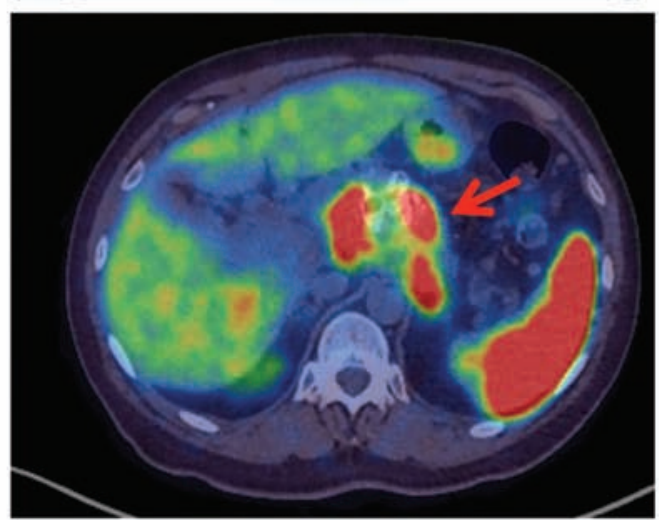

B

Fig 2. A patient with pancreatic, liver and skeletal metastases. A - ${ }^{68} \mathrm{Ga}$-DOTATATE maximal intensity projection image demonstrating pancreatic primary tumour (red arrow) with liver (orange arrow) and small volume skeletal metastases (blue arrow); B - ${ }^{177}$ Lu-DOTATATE post-therapy image showing the same biodistribution.

\section{Combination PRRT therapies}

Combination therapies have been used in PRRT, either combining different radionuclides (ie ${ }^{90} \mathrm{Y}$ and ${ }^{177}$ Lu-labelled peptides) or combining PRRT with radiosensitising chemotherapy.

Combination dual radiopeptide therapies have been performed as tandem therapies (ie administrating a combination of ${ }^{90} \mathrm{Y}$ and ${ }^{177} \mathrm{Lu}$-radiopeptides at a single sitting) or sequential therapies with ${ }^{90} \mathrm{Y}$ and ${ }^{177}$ Lu-radiopeptides. ${ }^{22,23}$ Combination PRRT have been shown to prolong survival over single agent PRRT. ${ }^{22,23}$ However, these studies were non-randomised retrospective reviews and there was potential selection bias in the groups.

Treatment with radiosensitising chemotherapy has also been reported to improve the success of PRRT, with disease response/stabilisation seen in $96 \%$ of patients. ${ }^{21}$

\section{Patient selection for PRRT in NETs}

The therapeutic options in NETs should be discussed in a multidisciplinary setting, with the choice of the most appropriate technique made. In the majority of patients surgical resection offers the only realistic possibility of cure and thus this should be considered the preferred option if curative surgery is feasible and the patient is fit enough.

In patients with unresectable metastatic disease there are various therapeutic options available. These include surgical debulking, chemotherapy, molecular targeted therapies (eg sunitinib and everolimus), interferon, somatostatin analogues, local therapies (eg radiofrequency ablation and embolisation) and radiotargeted therapies including PRRT.

The key component to patient selection for PRRT is the SSR scan. Sufficient uptake within tumours on the somatostatin receptor scintigraphy provides evidence that the tumours are likely to concentrate radioactivity in sufficient quantities to achieve tumour damage (theranostic principle). Based on SSR imaging, the liver is commonly used as the reference point (the liver having tracer uptake related to hepatic peptide metabolism). Uptake greater than that seen in the background liver in the majority of disease (ie $>90 \%$ ) is generally considered sufficient to proceed with PRRT.

Patient selection for PRRT in NETs includes patients who have histologically proven NET, who are SSR positive and have no surgical cure possible or patients who are unsuitable for surgery. Patients should have sufficient bone marrow reserve, ie platelets $>90,000 / \mu \mathrm{L}$ for ${ }^{90} \mathrm{Y}$ and $>75,000 / \mu \mathrm{L}$ for ${ }^{177} \mathrm{Lu} .{ }^{24}$ Additionally, significant renal impairment is a relative contraindication, particularly if ${ }^{90}$ Y-radiopeptides are being used.

Those with extensive disease, eg extensive liver/bone metastases, tend to have worse outcomes with PRRT. Similarly, it has been shown that significantly poorer outcomes occur in patients with poor baseline functional status. ${ }^{18}$ Grading of the tumour also has a role in treatment with PRRT, as patients with G3 tumours should have platinum-based chemotherapy rather than PRRT as first-line therapy.

FDG PET/CT may be useful in predicting response to PRRT and in determining progression-free survival, with those with 


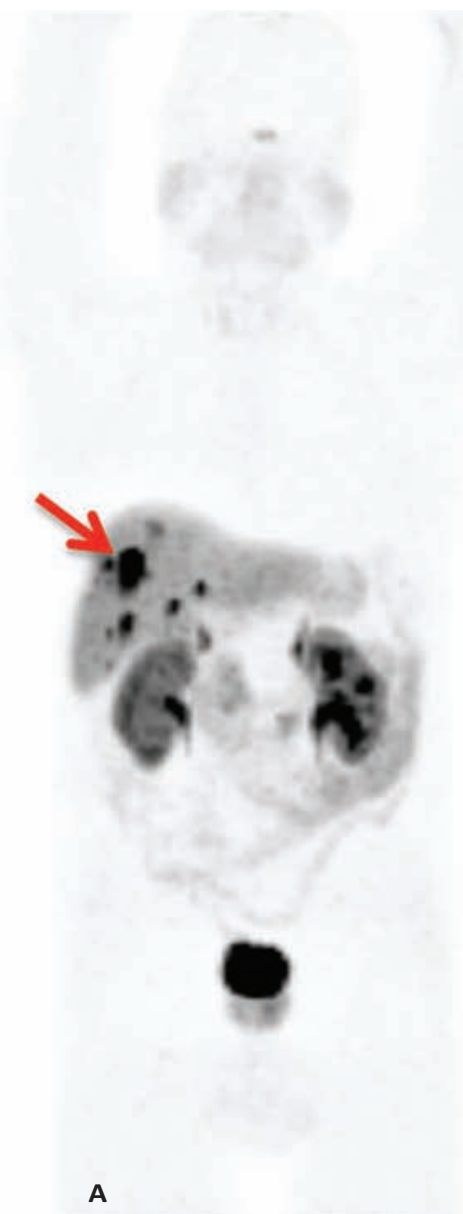

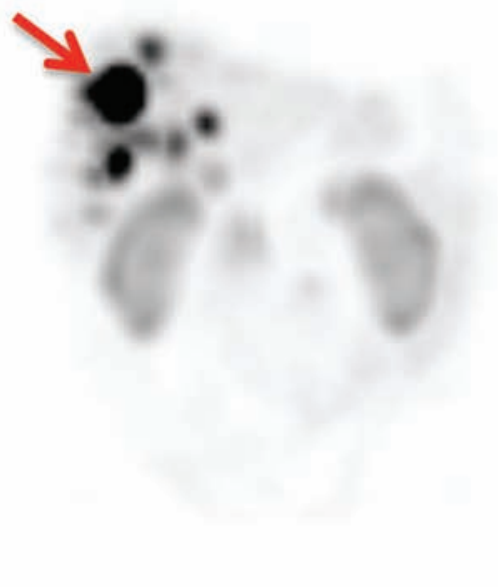

B

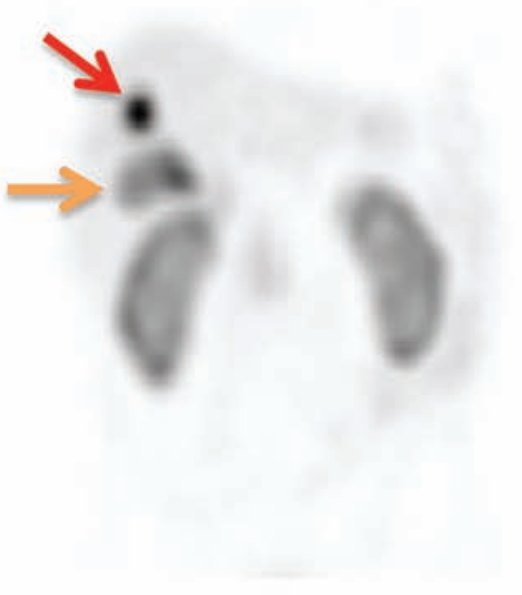

C

Fig 3. A patient with bilobar liver metastases. ${ }^{68} \mathrm{Ga}$-DOTATATE maximal intensity projection image demonstrates intense uptake in bilobar liver metastases (A). The red arrow points to a target liver lesion. The patient had ${ }^{177}$ Lu-DOTATATE therapy. Post-therapy planar ${ }^{177}$ Lu-DOTATATE studies performed after the first (B) and third (C) cycle of treatment demonstrate a significant reduction in the majority of liver lesions with the target liver lesion having significantly reduced uptake, consistent with a good response to treatment. The orange arrow represents physiological activity in the gallbladder.

FDG positive G1/G2 tumours having worse progression-free survival. $^{25}$

As NETs can be slow growing, the timing of treatment can be debatable. Patients with progressive disease or patients who are symptomatic despite cold somatostatin analogues should be the patients considered for therapy.

\section{Practical considerations of PRRT}

Approximately $50 \%$ of the radiopeptide is excreted in urine during the first 6-8 hours. Thereafter, minimal urinary excretion is seen and the main radiation protection issues are related to the low-abundance gamma rays emitted. Thus, patients are advised of standard radiation protection precautions, eg sleeping in a separate room to their partner, avoiding close contact with young children or pregnant women.

The majority of procedures are well tolerated and, in some centres in the UK, are performed as day cases. Care has to be taken in patients with functioning syndromes, eg VIPOMAs or symptomatic carcinoid syndrome, and thus it's safer to treat these patients as inpatients.

\section{MIBG imaging and therapy}

MIBG is an alkylguanidine (catecholamine analogue); it enters the cell membrane via the norepinephrine transporter and is then transported and stored in neurosecretory granules through the vesicular monoamine transporter. ${ }^{26-28}$ MIBG was first described for imaging of NETs in the late 1970s with ${ }^{131}$ I-MIBG. ${ }^{29}$ Radio-iodinated MIBG is well established in the detection of catecholamine-secreting neuroectodermal tumours (phaeochromocytoma and paraganglioma), with overall sensitivity around 90\%. MIBG imaging has also been used in NETs, with rates of positivity at around $70 \% .{ }^{4}$ In several studies comparing ${ }^{123} \mathrm{I}$-MIBG and ${ }^{111}$ In-pentetreotide, ${ }^{111} \mathrm{In}$ pentetreotide was found to be more sensitive for the detection of disease in NETs. ${ }^{30-32}$ The majority of NETs are thus not imaged/ staged with MIBG but with somatostatin receptor scintigraphy. Although MIBG imaging may not be useful in staging a NET patient, it may be useful in determining if the patient can be treated with ${ }^{131}$ I-MIBG radionuclide therapy - ie do all/majority of tumours have sufficient uptake to concentrate a $\beta$-emitting radiation within their tumours. ${ }^{33-35}{ }^{131}$ I-MIBG is a $\beta$-emitting radionuclide with the $\beta$ particle having a maximum range of 2.3 


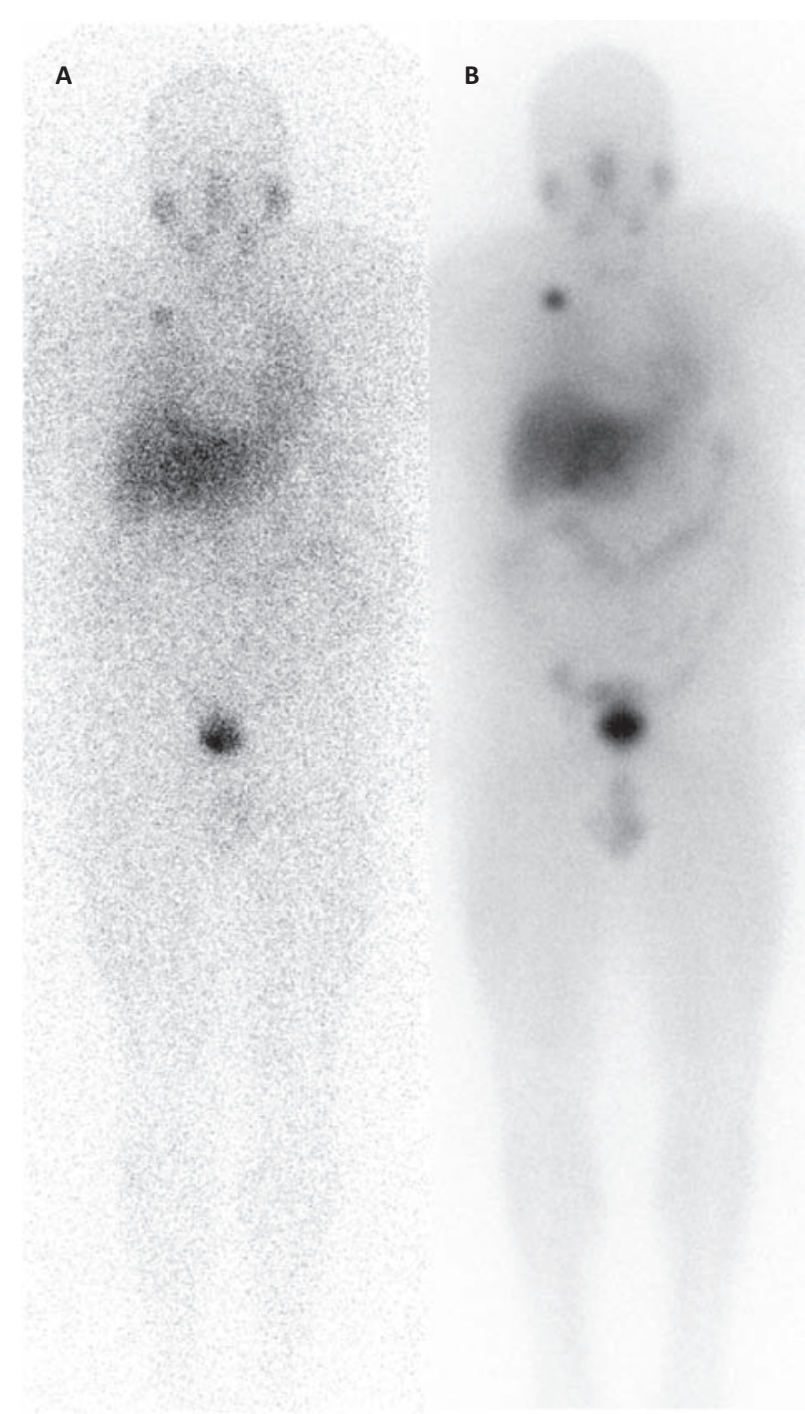

Fig 4. Anterior images of $a^{123}$ I-meta-iodobenzyl guanidine (MIBG) study (A) and ${ }^{131}$ I-MIBG post-therapy image (B). This patient with progressive metastatic midgut neuroendocrine tumour has uptake in their liver and right infraclavicular nodal metastases on ${ }^{123}$ I-MIBG study. They subsequently had ${ }^{131}$ I-MIBG therapy and had stable disease after three cycles.

$\mathrm{mm}$. This agent has been shown to stabilise disease in patients with progressive metastatic disease. An example of a patient with good uptake on the MIBG imaging scan that went on to have MIBG therapy is demonstrated in Fig 4.

Approximately $40-50 \%$ of patients develop good symptomatic response to treatment. ${ }^{33-35}$ Not many studies evaluated progression-free survival, but most studies showed a median overall survival of over 40 months following MIBG therapy. Interestingly, Sywak et al compared two groups of patients with midgut NETs. ${ }^{36}$ The first group $(n=58)$ was treated in a centre where ${ }^{131}$ I-MIBG was available while the second group $(\mathrm{n}=59)$ had no access to ${ }^{131}$ I-MIBG (or other radio-targeted treatments). The 5-year survival rate in the first group was $63 \%$ versus $47 \%$ in the second group $(\mathrm{p}=0.1)$

\section{${ }^{131}$ I-MIBG toxicity}

The main toxicities are bone marrow suppression and myelodysplasias. Grade 3/4 bone marrow toxicity was seen in approximately $8 \%$ of patients (range $2-25 \%$ ) with some relationship between the administered activity and the degree of toxicity. The most common form of bone marrow toxicity was thrombocytopenia (11\%) followed by leucopenia (10\%). Myelodysplasia is another possible rare side effect, which may occur in patients heavily pre-treated with chemotherapy or radiotherapy. ${ }^{33-35}$

\section{Tumour and critical organ dosimetry}

Dosimetry is the measurement of absorbed radiation dose imparted by ionising radiation. Dosimetry can be performed both to calculate critical organ radiation dose (to limit toxicity) and to optimise tumour dose. Critical organ activity-limiting toxicity is usually myelotoxicity and red marrow dosimetry is therefore of particular relevance. This is a challenging procedure that can be addressed by performing whole-body dosimetry as a surrogate measure, which can be derived accurately from external measurements (gamma camera whole-body imaging). ${ }^{37}$ Varying patient biokinetics lead to a wide range of reported absorbed whole-body doses. ${ }^{38,39}$ An even wider range of absorbed doses is delivered to tumours for all therapy procedures. Ilan et al found from three single photon emission CT scans tumour absorbed doses ranging from 10-340 Gy and found a significant correlation between the absorbed dose and tumour reduction. ${ }^{40}$

As personalised medicine and image-guided treatments (theranostics) are further introduced into clinical practice, dosimetry-based treatment is a promising avenue for further exploration via multicentre clinical trials to optimise the therapeutic procedure.

\section{Current status of PRRT in the UK}

Currently PRRT is not funded by NHS England. It was removed from the cancer drugs fund in November 2015 although funding for PRRT is still available through NHS Wales and NHS Scotland. Access to PRRT in England is principally limited to clinical trials and in a small number of patients on a compassionate use basis. Based on the NETTER-1 study, it is expected that ${ }^{177} \mathrm{Lu}$-DOTATATE will receive European marketing authorisation (EMA) later this year. Currently, the National Institute for Health and Care Excellence is appraising the use of ${ }^{177}$ Lu-DOTATATE, but formal guidance will only be published after EMA approval.

\section{Conclusions}

Nuclear medicine imaging and therapy plays a vital role in management of NETs. SSR imaging provides enhanced accuracy in staging with accuracies of $>90 \%$ in well differentiated tumours and allows change in management compared with conventional imaging. Radionuclide therapies with radiolabelled somatostatin analogues and radio-iodinated MIBG provide symptomatic benefit and increase survival in patients with metastatic NETs. It is in NETs that the theranostic concept first became popularised.

\section{Conflicts of interest}

The authors have no conflicts of interest to declare. 


\section{References}

1 Ramage JK, Ahmed A, Ardill J et al. Guidelines for the management of gastroenteropancreatic neuroendocrine (including carcinoid) tumours (NETs). Gut 2012;61:6-32.

2 Rindi G, Kloppel G, Couvelard A et al. TNM staging of midgut and hindgut (neuro) endocrine tumors: a consensus proposal including a grading system. Virchows Arch 2007;451:757-62.

3 Baum RP, Kulkarni HR, Carreras C. Peptides and receptors in image-guided therapy: theranostics for neuroendocrine neoplasms. Semin Nucl Med 2012;42:190-207.

4 Rufini V, Calcagni ML, Baum RP. Imaging of neuroendocrine tumours. Semin Nucl Med 2006;36:228-47.

5 Teunissen JJM, Kwekkeboom DJ, de Jong M et al. Peptide receptor radionuclide therapy. Best Pract Res Clin Gastroenterol 2005;19:595-616.

6 Reubi JC. Regulatory peptide receptors as molecular targets for cancer diagnosis and therapy. Q J Nucl Med 1997;41:63-70.

7 Krenning EP, Bakker WH, Breeman WA et al. Localisation of endocrine-related tumours with radioiodinated analogue of somatostatin. Lancet 1989;1:242-4.

8 Decristoforo C, Melendez-Alafort L, Sosabowski JK, Mather SJ. 99mTc- HYNIC-[Tyr3]-octreotide for imaging somatostatinreceptor-positive tumors: preclinical evaluation and comparison with 111In-octreotide. J Nucl Med 2000;41:1114-9.

9 De Herder WW Kwekkeboom DJ, Valkema R et al. Neuroendocrine tumors and somatostatin: imaging techniques. J Endocrinol Invest 2005;28:132-6.

10 Pettinato C, Sarnelli A, Di Donna M et al. 68Ga-DOTANOC: biodistribution and dosimetry in patients affected by neuroendocrine tumors. Eur J Nucl Med 2008;35:72-9.

11 Antunes $\mathrm{P}$, Ginj M, Zhang $\mathrm{H}$ et al. Are radiogallium-labelled DOTA-conjugated somatostatin analogues superior to those labelled with other radiometals? Eur J Nucl Med 2007;34:982-93.

12 Srirajaskanthan R, Kayani I, Quigley AM et al. The role of 68Ga-DOTATATE PET in patients with neuroendocrine tumors and negative or equivocal findings on 111In-DTPA-octreotide scintigraphy. J Nucl Med 2010;51:875-82.

13 Breiner Geijer H. Somatostatin receptor PET/CT in neuroendocrine tumours: update on systematic review and meta-analysis Eur J Nucl Med 2013;40:1770-80.

14 Imhof A, Brunner P, Marincek N et al. Response, survival, and longterm toxicity after therapy with the radiolabeled somatostatin analogue [90Y-DOTA]-TOC in metastasized neuroendocrine cancers. J Clin Oncol 2011;29:2416-23.

15 Bushnell DLJr, O’Dorisio TM, O’Dorisio MS et al. 90Y-edotreotide for metastatic carcinoid refractory to octreotide. J Clin Oncol 2010;28:1652-9.

16 Cwikla JB, Sankowski A, Seklecka N et al. Efficacy of radionuclide treatment DOTATATE Y-90 in patients with progressive metastatic gastroenteropancreatic neuroendocrine carcinomas (GEP-NETs): a phase II study. Ann Oncol 2010;21:787-94.

17 Toumpanakis C, Quigley A, Srirajaskanthan R. 90-Yttrium-DOTAoctreotate for the treatment of advanced neuroendocrine tumors. J Clin Oncol 2009;27 (Suppl 15):4594.

18 Kwekkeboom DJ, de Herder WW, Kam BL et al. Treatment with the radiolabeled somatostatin analog [177Lu-DOTA0,Tyr3] octreotate: toxicity, efficacy, and survival. J Clin Oncol 2008;26:2124-30.

19 Pencharz D, Walker M, Yelchin M et al. Early efficacy of and toxicity from lutetium-177-DOTATATE treatment in patients with progressive metastatic NET. Nucl Med Commun 2017;38:593-600.

20 Strosberg J, El-Haddad G, Wolin E et al. Phase 3 trial of 177Lu-Dotatate for midgut neuroendocrine tumors. N Engl J Med 2017;376:125-35.

21 Kunikowska J, Królicki L, Hubalewska-Dydejczyk A et al. Clinical results of radionuclide therapy of neuroendocrine tumours with 90Y-DOTATATE and tandem 90Y/177Lu-DOTATATE: which is a better therapy option? Eur J Nucl Med Mol Imaging 2011;38:1788-97.

22 Villard L, Romer A, Marincek N et al. Cohort study of somatostatin-based radiopeptide therapy with [90Y-DOTA]-TOC versus
[90Y-DOTA]-TOC plus [177Lu-DOTA]-TOC in neuroendocrine cancers. J Clin Oncol 2012;30:1100-6.

23 Claringbold PG, Brayshaw PA, Price RA et al. Phase II study of radiopeptide $177 \mathrm{Lu}$-octreotate and capecitabine therapy of progressive disseminated neuroendocrine tumors. Eur J Nucl Med 2011;38:302-11.

24 Zaknun JJ, Bodei L, Mueller-Brand J et al. The joint IAEA, EANM, and SNMMI practical guidance on peptide receptor radionuclide therapy (PRRNT) in neuroendocrine tumours Eur J Nucl Med 2013;40:800-16.

25 S1 Severi, O Nanni, Bodei L et al. Role of 18FDG PET/CT in patients treated with 177Lu-DOTATATE for advanced differentiated neuroendocrine tumours Eur J Nucl Med 2013;40:881-8.

26 Wieland DM, Brown LE, Tobes MC. Imaging the primate adrenal medulla with 123I and 131I metaiodobenzylguanidine. Concise communication. J Nucl Med 1981;22:358-64.

27 Solanki KK, Bomanji J, Moyes J et al. A pharmacological guide to medicines which interfere with the biodistribution of radiolabelled meta-iodobezylguanidine (MIBG). Nucl Med Comm 1992;13:513-21.

28 van der Harst E, de Herder WW, Bruining HA et al. [(123)I]metaiodobenzylguanidine and [(111)In] octreotide uptake in benign and malignant pheochromocytomas. J Clin Endocrinol Metab 2001;86:685-93.

29 Wieland DM, Swanson DP, Brown LE, Beierwaltes WH. Imaging the adrenal medulla with an I-131-labeled antiadrenergic agent. J Nucl Med 1979;20:155-8.

30 Ramage JK, Williams R, Buxton-Thomas M. Imaging secondary neuroendocrine tumours of the liver: comparison of I123 metaiodobenzylguanidine (MIBG) and In111-labelled octreotide (Octreoscan). QJM 1996;89:539-42.

31 Kaltsas G, Korbonits M, Heintz E et al. Comparison of somatostatin analog and metaiodobenzylguanidine radionuclides in the diagnosis and localization of advanced neuroendocrine tumours. J Clin Endocrinol Metab 2001;86:895-902.

32 Nocaudie-Calzada M, Huglo D, Carnaille B, Proye C, Marchandise $\mathrm{X}$. Comparison of somatostatin analogue and metaiodobenzylguanidine scintigraphy for the detection of carcinoid tumours. Eur J Nucl Med 1996;23:1448-54.

33 Safford SD, Coleman RE, Gockerman JP et al. Iodine-131 metaiodobenzylguanidine treatment for metastatic carcinoid. Results in 98 patients Cancer 2004;101:1987-93.

34 Nwosu AC, Jones L, Vora J et al. Assessment of the efficacy and toxicity of 131I-metaiodobenzylguanidine therapy for metastatic neuroendocrine tumours Br J Cancer 2008;98:1053-8.

35 Navalkissoor S, Alhashimi DM, Quigley AM, Caplin ME, Buscombe JR. Efficacy of using a standard activity of (131)I-MIBG therapy in patients with disseminated neuroendocrine tumours. Eur J Nucl Med 2010;37:904-12.

36 Sywak MS, Pasieka JL, McEwan A, Kline G, Rorstad O. 131I- metaiodobenzylguanidine in the management of metastatic midgut carcinoid tumors. World J Surg 2004;28:1157-62.

37 Chittenden SJ, Pratt BE, Pomeroy K et al. Optimization of equipment and methodology for whole-body activity retention measurements in children undergoing targeted radionuclide therapy. Cancer Biother Radiopharm 2007;22:243-9.

38 Monsieurs M, Brans B, Bacher K, Dierckx R, Thierens HT. Patient dosimetry for 131I-MIBG therapy for neuroendocrine tumours based on 123I-MIBG scans. Eur J Nucl Med 2002;29:1581-7.

39 Flux GD, Chittenden SJ, Saran F, Gaze MN. Clinical applications of dosimetry for mIBG therapy. Q J Nucl Med Mol Imaging 2011;55:116-25.

40 Ilan E, Sandström M, Wassberg C et al. Dose response of pancreatic neuroendocrine tumors treated with peptide receptor radionuclide therapy using 177Lu-DOTATATE. J Nucl Med 2015;56:177-82.

Address for correspondence: Dr Shaunak Navalkissoor, Department of Nuclear Medicine, Royal Free London NHS Foundation Trust, Pond Street, Hampstead, London NW3 2QG, UK.

Email: s.navalkissoor@nhs.net 